\title{
Warping source deghost in multicomponent post-stack data
}

\section{CJ Calderon Filho $\left(^{*}\right)$ - PETROBRAS}

Copyright 2019, SBGf - Sociedade Brasileira de Geofísica

This paper was prepared for presentation during the $16^{\text {th }}$ International Congress of the Brazilian Geophysical Society held in Rio de Janeiro, Brazil, 19-22 August 2019.

Contents of this paper were reviewed by the Technical Committee of the $16^{\text {th }}$ International Congress of the Brazilian Geophysical Society and do not necessarily represent any position of the SBGf, its officers or members. Electronic reproduction or storage of any part of this paper for commercial purposes without the written consent of the Brazilian Geophysical Society is prohibited.

\section{Abstract}

For a multicomponent data, it is possible to combine the hydrophone and geophone records to attenuate the receiver ghost, but the source ghost would still be present on the data. If the source ghost is not addressed on the processing steps prior to migration and stacking, that is, if only a phase correction is applied to address the source ghost the amplitude spectrum will still contain associated notches. In this work we compare a spectral balancing with a warping source deghost to solve the problem above. As we will see, the latter result has more continuity between events and more low frequency content.

\section{Introduction}

The sources and receivers for marine acquisition are placed a few meters deep instead of at the water surface for theoretical and practical purposes. Therefore, the data suffers a reduction of its useful bandwidth due to the presence of ghost reflections.

Several techniques were created to attenuate the ghost effect since the 1950s [Haggerty, 1956]. Multicomponent acquisitions mitigate the receiver ghost with a combination of hydrophone and vertical accelerometer data, but the source ghost still needs to be attenuated by seismic processing.

There are some multicomponent data that have been migrated and stacked in which the amplitude source ghost is still present, although the data is in zero phase and therefore without the phase source ghost. One simple solution to increase the bandwidth in this case is a spectral balancing, but a better solution can be achieved if we add the phase source ghost again in the data and use a robust deghost method.

For this work, we use a warping deghost method [Filpo, 2017]. In this technique, each ghost component present in the data is a deformed version of the signal free of ghosts. This idea is valid on the hypothesis of the absence of the direct wave and general external noises. Besides that, the source depth is known throughout the whole survey, as well as the velocity model and the reflection coefficient on the water-air interface.

\section{Theory}

The fact that ghost reflections are deformed versions of the original signal suggests that possible transformations between them can be done by warping algorithms [Beasley, 2013]. In this way, the registered signal $y(t)$ can be written as:

$$
y(t)=s(t)-\alpha(t) s(t+\mu(t))
$$

where $s(t)$ is the original signal without any reflection ghost, $\alpha$ is a scale factor and $\mu$ is a time shift between the primary and the ghost reflection. In matrix notation:

$$
\boldsymbol{y}=(\boldsymbol{I}-\boldsymbol{W}) \boldsymbol{s}
$$

where $\boldsymbol{W}$ is the warping matrix. We can decompose $\boldsymbol{W}$ as follows:

$$
y=\left(I-W_{s}-W_{r}+W_{s r}\right) s
$$

where $\boldsymbol{W}_{\boldsymbol{s}}, \boldsymbol{W}_{\boldsymbol{r}}$ and $\boldsymbol{W}_{\boldsymbol{s} r}$ are the warping operators that perform the distortions related to the source, receiver and source-receiver ghosts, respectively. The previous equation can be rewritten as:

$$
y=A s
$$

Thus the solution of this equation system is:

$$
s=\left(A^{T} A\right)^{-1} A^{T} y
$$

or, iteratively:

$$
s^{(n+1)}=s^{(n)}-\beta A^{T}\left(A s^{(n)}-y\right)
$$

where $\beta$ is the step parameter.

The $\boldsymbol{A}$ matrix is easily obtained by ray tracing techniques if the velocity model is known, as well as the receiver and the source depths.

\section{Results}

A PSDM post-stack broadband data was used to test the warping deghost. This data was obtained in a combination of the hydrophone record and the vertical component of the geophone record. Because of that, the receiver ghost was attenuated and only the source ghost remained. However, this data was set in zero phase on a processing step before the depth migration and therefore the phase of source ghost operator is not present.

To address this problem, two solutions were tested: (1) a spectral balancing on the 04-60 Hz bandwidth and (2) reapplying the phase of the source ghost operator, applying a warping deghost and then an amplitude $Q$ Compensation to increase the spectrum. Before these two tests, the PSDM data was converted to time domain. 
In Figure 1.a, we show a water bottom zoom on the original PSDM data in time domain. It is possible to observe that the data was in zero phase and then the deghost operators would add some non-zero phase if applied on this data directly. In Figure 1.b, the phase of source ghost was added and in Figure 1.c we can see the result after the warping deghost. It is possible to see that the result is also in zero phase. One important thing to note is that the side lobes present on the data in Figure 1.a is attenuated in Figure 1.c. One can argue that we have a better resolution in Figure 1.a than Figure 1.c, and it is compatible with the spectra in Figure 5. However, that high frequency content above $80 \mathrm{~Hz}$ in the original data is only present near the water bottom and do not compromise the results on the deeper part.

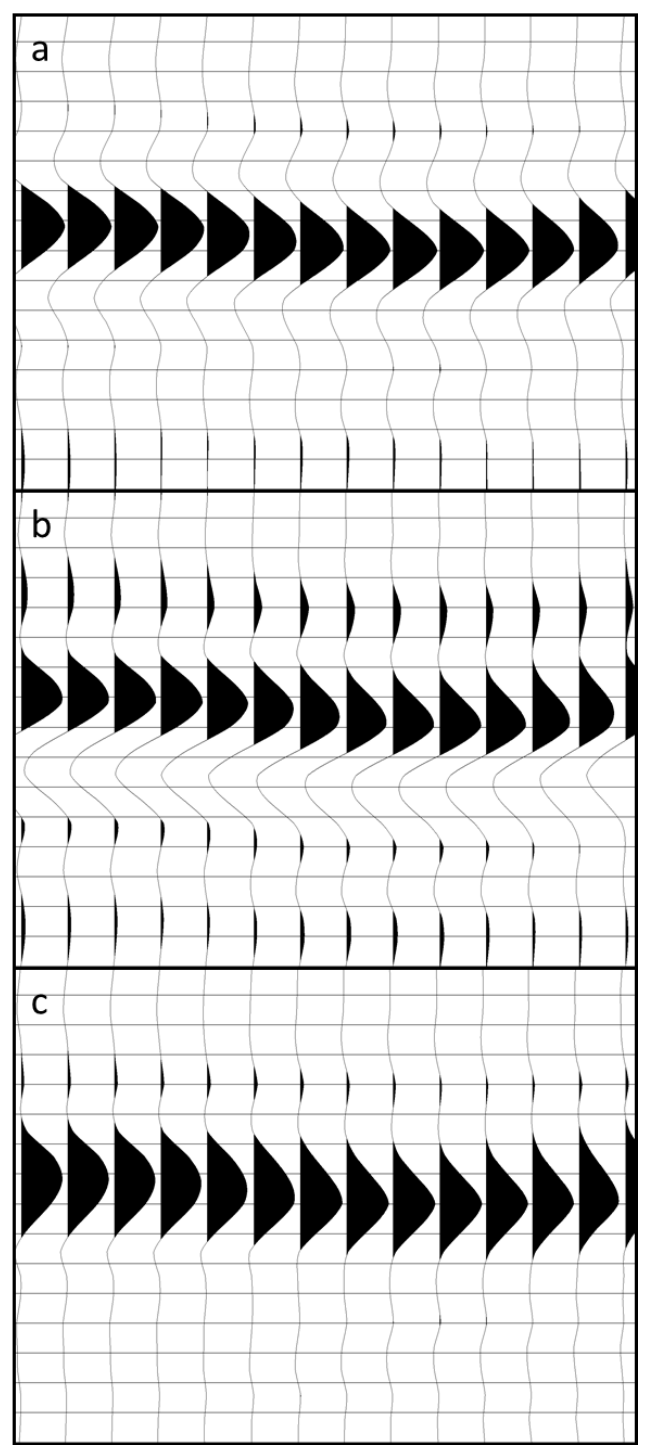

Figure $1-A$ zoom of the water bottom: (a) the original PSDM data converted to time domain, (b) the result of adding the phase of source ghost, (c) the result after the warping deghost.
We can compare the original PSDM data (figure 2) with the result of the warping deghost (figure 3 ) and the result with only the spectral balancing (figure 4). It is possible to see that we gain resolution with both results, but we have better continuity of the events and low frequency content in the warping deghost one.

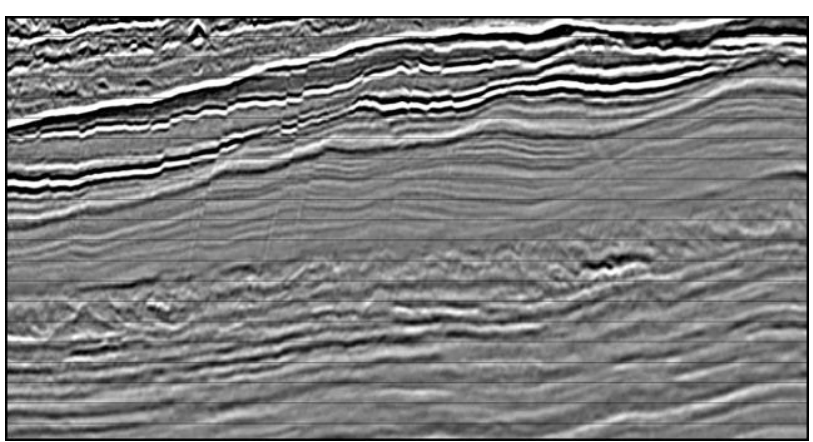

Figure 2 - The original PSDM data converted to time domain.

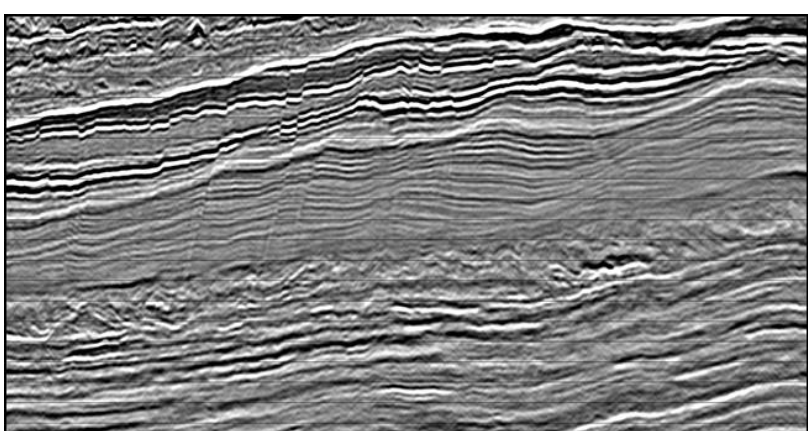

Figure 3 - The result after the warping deghost. Here we put again the phase of the source ghost operator and then applied the warping deghost. To increase the final resolution, we applied an amplitude $Q$ compensation.

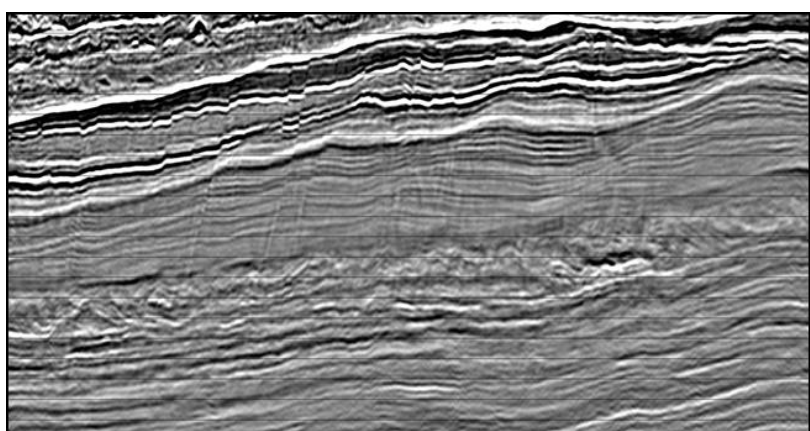

Figure 4 - The result after the application of the spectral balancing. 
In figure 5, we can compare the amplitude spectra of figures 2, 3 and 4 . As one can see, we have a better low frequency content on the warping deghost one and comparable high frequency content.

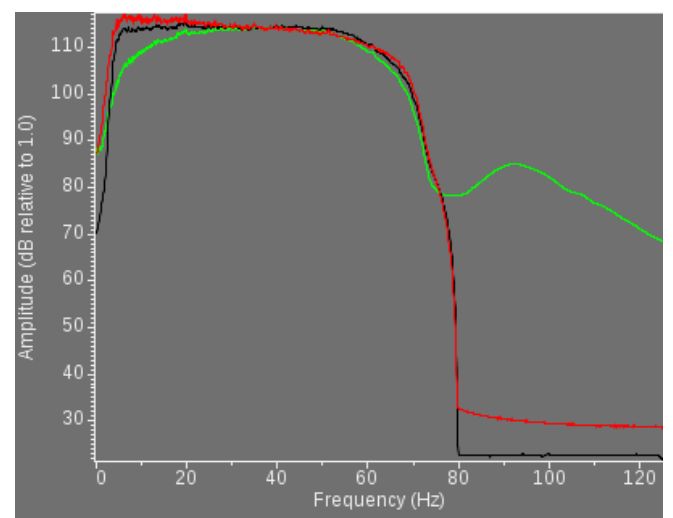

Figure 5 - Amplitude spectra of the results in $d B$. The original PSDM data is in green, the warping deghost is in red and the spectral balancing is in black.

\section{Conclusions}

With the increasing interest in multicomponent data, it is important to know how to better deal with the ghost. Some combinations of this kind of data attenuate the receiver ghost but the source ghost would still be present. In some cases the amplitude source ghost is still present in the stacked data but not the phase of this operator.

There are many solutions for the problem above, considering the solutions addressed in this paper we found that the most robust is to put the phase of the source ghost back to the data and then applying a deghost algorithm. For this case, we use a warping deghost because of the simplicity of the algorithm and the quality obtained in previous results [Filpo, 2014, 2015, 2017].

When we compare the results obtained with the warping deghost with the results where only a spectral balancing was applying (figures 3 and 4, respectively), we see more continuity of the events and more low frequency content in the latter.

\section{Acknowledgments}

The author would like to thank PETROBRAS for authorizing this publication. The author would like to thank Eduardo Filpo, Gilberto Lima, Carlos Alves da Cunha Filho and André Gomes de Araujo for the insightful ideas and Ricardo Felipe Chartuni Cabral da Cruz for all help and support to use the warping deghost algorithm.

\section{References}

Beasley, C. J., Coates, R., Ji, Y. and Perdomo, J., 2013. Wave equation receiver deghosting: a provocative example: Expanded Abstracts, SEG 83 ${ }^{\text {rd }}$ Annual International Meeting, 32: 4226-4230.

Filpo, E. and Lima, G., 2014. Deghosting via warping: $2^{\text {nd }}$ EAGE/SBGF Workshop - Broadband Seismic Expanded Abstracts.

Filpo, E., Lima, G. and Lobo, P., 2015. On the application of warping deghosting: $14^{\text {th }}$ International Congress of SBGf Expanded Abstracts.

Filpo, E., Lima, G. and Anibal, M., 2017. Improving seismic resolution via warping deghosting: $15^{\text {th }}$ International Congress of SBGf Expanded Abstracts.

Haggerty, P. E., 1956. Method and apparatus for canceling reverberations in water layers: U.S. Patent 2,757,356. 